\title{
Morfología de los Adjetivos Demostrativos en el Rromané Jorajané de Chile ${ }^{1}$
}

\author{
Diego Gonzalo Lizarralde Contreras* \\ Gastón Felipe Salamanca Gutiérrez ${ }^{* *}$
}

\section{Resumen}

Este trabajo, dividido en dos artículos, presenta una descripción en el plano morfológico para una lengua poco estudiada en el ámbito nacional: el rromané jorajané hablado por los gitanos de Chile.

Este primer artículo contiene una introducción etnográfica -donde se presentan unas notas breves sobre los aspectos más prominentes de la lengua de los gitanos-, los antecedentes de la investigación, los objetivos generales y específicos planteados, el marco de referencia y metodología utilizados, y, como foco, un análisis morfológico de los demostrativos. Este último punto contiene una nota introductoria sobre el tratamiento de los demostrativos en la gramática castellana, un cuadro con las formas de los demostrativos en rromané de Chile, una propuesta de segmentación morfémica, y una propuesta de asignación de morfos a morfemas. A cada uno de los puntos recién mencionados, le siguen observaciones que abordan aspectos morfológicos, fonémicos y comparativos respecto del español.

Palabras clave: Gitanos, rromané, demostrativos en rromané.

\begin{abstract}
This study, divided into two articles, is a description of the morphology of a language that has drawn little attention nationally: Rromané Jorajané, spoken by Gypsies in Chile. This first article contains an introduction to Gypsy ethnography -which includes some brief notes regarding the most prominent features of the language-; earlier research; proposed general and specific objectives; the theoretical framework and methodology employed; and, as a focal point, the morphological analysis of demonstrative adjectives. This final point includes an introductory note regarding the treatment of demonstrative adjectives in Spanish grammar, a chart of demonstrative adjective forms in Chilean Rromané, a proposal for morphemic segmentation, and a proposal for assigning morphs to morphemes. Observations regarding morphological, phonemic, and comparative aspects with respect to Spanish follow each of the foregoing points.
\end{abstract}

Keywords: Gypsies, Rromané, demonstrative adjectives in Rromané.

1 Esta investigación viene a continuar los trabajos iniciados por González y Salamanca (2001) sobre la fonología del rromané y la tesis doctoral de Salamanca (2002) sobre la morfología nominal y verbal de esta lengua. A lo anterior se suma nuestra propuesta grafémica (Salamanca y Lizarralde, 2008), la cual es utilizada aquí para la escritura de las palabras en rromané (escritas en cursiva).

* Doctorando en Lingüística. Universidad de Concepción. dlizarra@udec.cl

** Doctor en Lingüística, Universidad de Concepción. gaston.salamanca@gmail.com 


\section{Introducción}

\subsection{Consideraciones generales}

El trabajo que presentamos se inscribe dentro de la tradición de la lingüística descriptiva, en un área de amplio desarrollo en esta línea, como es la descripción de lenguas vernáculas o minoritarias². Proponemos una descripción en el plano morfológico para una lengua muy poco estudiada en el ámbito nacional: el rromané jorajané hablado por los gitanos de Chile.

El presente artículo, junto con el que le sigue ("Morfología de los adjetivos posesivos en el Rromané Jorajané de Chile", de pronta publicación), son el resultado de una investigación unitaria llevada a cabo por los autores; el marco teórico y metodológico es, entonces, el mismo para ambos trabajos, razón por la cual hemos decidido incluir estos apartados solamente en esta primera comunicación. Esto explica que en el trabajo sobre los posesivos el análisis morfológico se presente de manera inmediata. Por su parte, dada la unidad entre ambos trabajos, las conclusiones se presentan al final del segundo artículo y en ellas se destacan los puntos más relevantes del análisis de ambas subclases de palabras (adjetivos demostrativos y posesivos) y las líneas de proyección de esta investigación.

En el contexto de los escasos estudios relativos a la lengua hablada por los gitanos de Chile (ver punto 2), nos parece que el presente trabajo aborda un objeto de estudio novedoso y constituye un aporte a la descripción lingüística, pues es la primera publicación sobre la morfología de los adjetivos del rromané jorajané hablado en Chile. Por esto mismo, el trabajo se presenta como una contribución relevante al estudio de esta lengua en particular y un paso adelante en el objetivo de la elaboración de una gramática del rromané hablado por los gitanos de nuestro país.

\subsection{Notas Etnolingüísticas}

\subsubsection{El panrromani}

Si se considera el grado de retención de la lengua original, se pueden distinguir tres grandes grupos de gitanos:

a) Los que mantienen un alto grado de retención del rromaní, con diferencias dialectales. Tal es el caso de los rrom de Chile y USA.

b) Los que sólo conservan una base léxica rromaní, como el caló en España.

2 Usamos el término legua vernácula en la medida en que el rromané jorajané puede ser considerado como "la lengua materna de un grupo dominado social o políticamente por otro que habla una lengua diferente" (UNESCO, citado en Wagner et all, 1978, pág. 62).

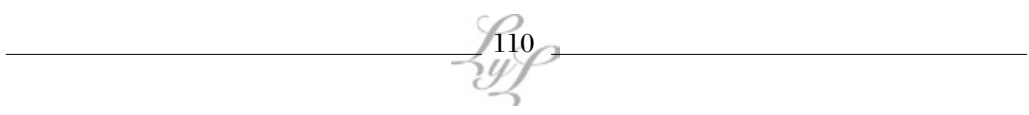


c) Aquellos que han perdido todo conocimiento del rromaní, como el grupo ludár de Chile y Argentina.

Uno de los rasgos característicos del panrromaní, en el plano fonológico, es la presencia de consonantes aspiradas (/ph, th, kh/), en contraste con las no aspiradas $(/ \mathrm{p}, \mathrm{t}, \mathrm{k} /)$. En el plano morfológico, destaca la expresión de las categorías gramaticales de género (masculino y femenino), número (singular y plural) y caso (nominativo, acusativo, dativo, ablativo, locativo, instrumental, genitivo y vocativo), a través de sufijos.

Otro de los rasgos característicos del panrromaní, y en el que coinciden los lingüistas dedicados al estudio de la lengua gitana (Hancock 1993, Grant 1994, Salamanca 2004b), es la división del estudio de la morfología de esta lengua en dos planos: morfología de ítemes tradicionales (denominada también "temática") y morfología de préstamos introducidos en épocas recientes (o "atemática"). En este sentido, Hancock señala que:

One of the most interesting characteristics of Romani is that it has two sets of grammatical rules: one for its Asian component, called thematic, and one for its European component, called athematic. The thematic rules apply to all the words from languages up to and including Byzantine Greek; the athematic rules apply, broadly speaking, to everything acquires from Balkan Greek onwards. The grammar for the thematic component is mainly Indian, and very regular, while the grammar for the athematic part of the language is more complex. For this reason, it isn't entirely accurate to call Romani a wholly Indian language; it seems to have finished taking shape only during the period of its contact with Greek, and so has a 'Balkan' character as well.

Thematic vocabulary is common to all Romani dialects, and it is the athematic loanwords from other languages that make one dialect different from another. As a dialect loses more and more of its original thematic vocabulary and replaces it with foreign adoptions, so it becomes less and less easily understood by speakers of other dialects. (Hancock, 2003: 150)

\subsubsection{El rromané hablado en Chile}

En Chile, los gitanos rrom llaman a su lengua rromané o rromanó. La denominación más frecuente es rromané. El rromané se distingue del (ga)llikané (lengua de los gallé = personas no gitanas) y del ludár -o boyás- (lengua hablada por algunos gitanos no rrom que viven en Santiago de Chile).

La interacción entre los gitanos del grupo rrom y los gitanos del grupo ludár se produce sólo en español, pues sus respectivas lenguas no son mutuamente inteligibles (Salamanca y González, 1999: 150). 
Los estudios lingüísticos que existen sobre el rromané jorajané de Chile abarcan los niveles fonológico y morfológico. Respecto del primero, González y Salamanca establecen que los fonemas segmentales del rromané de Chile son los siguientes: /i, e, a, o, u, p, b, ph, t, d, th, k, g, kh, f, v, s, z, x, č, dž, čh, m, n, ñ, 1, r, rr, j, w/ (González y Salamanca, 2001: 178 y sigtes.). Respecto del nivel morfológico, la investigación de Salamanca (2002) permite comprobar que las categorías gramaticales expresadas morfológicamente en el rromané jorajané de Chile coinciden con las mencionadas respecto del panrromané, esto es, género (masculino y femenino), número (singular y plural) y caso (nominativo, acusativo, dativo, ablativo, locativo, instrumental, genitivo y vocativo). En cuanto al verbo, el autor establece que el rromané de Chile tiene dos tiempos básicos: presente y pretérito perfecto, y tres tiempos derivados de los anteriores: futuro, pretérito imperfecto y pretérito pluscuamperfecto. Todos los tiempos verbales se marcan por medio de sufijos; excepto el futuro, el cual se marca a través de la llamada "partícula de futuridad".

El bilingüismo rromané-español de los gitanos rrom de Chile presenta dos características bien definidas: en primer lugar, existe un bilingüismo en el que el español está subordinado al rromané; y, en segundo lugar, existe entre los gitanos rrom una actitud muy positiva hacia su lengua. Ellos utilizan exclusivamente el rromané al interactuar con los miembros de la comunidad gitana rrom, y usan el español sólo cuando interactúan con algún galló o algún gitano boyás.

Después de examinar aspectos culturales y lingüísticos, Salamanca (2005), destaca la alta vitalidad de la cultura y de la lengua gitana, lo que es notable, sobre todo si lo consideramos en el contexto de una tendencia a la aculturación y la pérdida de la lengua que presentan las etnias vernáculas presentes en Chile, y el reducido número de personas que conforman el pueblo gitano (no más del 0,031\% de la población chilena).

\section{Antecedentes de la Investigación}

A diferencia de la situación de Europa y Norteamérica, en Sudamérica la etnia gitana no ha despertado mayor interés en las ciencias sociales en general y en la lingüística en particular. En Chile, las publicaciones sobre el tema no pasan del artículo periodístico de corte costumbrista. En este contexto, las investigaciones de González y Salamanca (2001) sobre la fonología del rromané y los trabajos de Salamanca (2002, 2004b, 2006 y 2008) sobre la morfología nominal y verbal de esta lengua constituyen trabajos pioneros en el tema gitano, que abren un nicho investigativo novedoso y de amplias proyecciones.

De acuerdo con el trabajo de Salamanca (2002), los sustantivos temáticos en el rromané jorajané hablado en Chile se construyen de acuerdo a la siguiente fórmula tagmémica: un casillero "núcleo nominal" obligatorio se instancia por un tema nominal, y cuatro casilleros marginales de "género", "caso complementario", "número" y "caso" se instancian por morfemas que indican estas 
funciones. Los casilleros marginales obligatorios son los de número y caso. El casillero de género se instancia en los sustantivos animados, cuando es posible un contraste. La fórmula tagmémica general es:

\begin{tabular}{|c|c|c|c|c|}
\hline Núcl. Nom. & Género & Caso Complem. & Número & Caso \\
\hline tema nom. & morf. De gén. & SICC & morf. de núm. & morf. de caso \\
\hline
\end{tabular}

Un ejemplo de la extensión máxima de esta fórmula es la palabra "rromiénchar" ('con las gitanas'). Aquí, el núcleo nominal es instanciado por el tema nominal "rrom-" ('persona que pertenece a la etnia gitana'); el casillero de género lo instancia el sufijo "-i" ('femenino'); el casillero de caso complementario es instanciado por el sufijo "-é" ('Sufijo Indicador de Caso Complementario'); el casillero de número lo instancia el sufijo "-n" ("plural'); y el casillero de caso gramatical es instanciado por el sufijo "-char" ('instrumental').

El tema nominal contiene un casillero "centro" obligatorio, instanciado por una raíz sustantiva o adjetiva, y un casillero "derivación" instanciado por un sufijo derivacional:

\begin{tabular}{|c|c|}
\hline Centro & Derivación \\
\hline Raíz adjetiva/raíz sustantiva & sufijo derivacional \\
\hline
\end{tabular}

Por ejemplo, el tema nominal "barbalipé" ('riqueza') contiene una raíz adjetiva "barbal-" ('que posee riqueza') que instancia el casillero "centro", y un sufijo "-ipé" que instancia el casillero de "derivación".

Otros antecedentes de particular utilidad para este trabajo fueron la Grammar of Vlax Romani (Hancock, 1993) y el trabajo de Franzese (1997) "Il dialetto dei rom xoraxané".

\section{Objetivos}

\section{Objetivo General}

- Describir la estructura interna de los adjetivos demostrativos en el rromané jorajané hablado por los gitanos de Chile.

\section{Objetivos Específicos}

- Identificar morfemas y alomorfos que indican la flexión de los adjetivos demostrativos.

- Identificar el orden de los morfemas en este tipo de adjetivos.

- Determinar los fenómenos morfofonémicos presentes en la flexión de los demostrativos del rromané jorajané. 


\section{Marco de Referencia}

El trabajo que presentamos toma como marco de referencia el modelo tagmémico postulado por Pike en la década de los cincuenta. La propuesta original de la tagmémica fue luego desarrollada por Elson y Pickett en los textos An Introduction to Morphology and Syntax (1964) y Beginning Morphology and Sintax (1980), los cuales son nuestra referencia directa. Señalamos esto porque en trabajos posteriores de los autores mencionados, encontramos desarrollos que muestran algunas diferencias respecto de esta versión clásica del modelo3 ${ }^{3}$.

La tagmémica, más que un marco teórico propiamente tal, es un modelo descriptivo, cuya rentabilidad está ampliamente demostrada en el ámbito de la lingüística descriptiva. Cuatro principios fundamentales de esta perspectiva, y que hemos seguido aquí, son los siguientes:

a) El lenguaje presenta constituyentes susceptibles de ser segmentados

b) Las unidades existen en función de su relación con otras unidades

c) Las unidades se organizan dentro de un sistema mayor de relaciones

d) El marco de referencia para describir las unidades debe ser flexible

En cuanto a la aplicación del modelo tagmémico, la tesis doctoral de Salamanca Morfología nominal y verbal del romané, lengua de los gitanos de Chile (2002) constituye una guía de trabajo que hemos seguido de cerca ${ }^{4}$.

En términos epistemológicos, basamos nuestro trabajo de descripción en los postulados del empiricismo funcional propuesto por Paul Garvin en su trabajo "Una epistemología empiricista para la lingüística" (1979). Los planteamientos de Garvin sustentan también, en buena medida, las decisiones metodológicas de nuestra investigación.

\section{Metodología}

\subsection{Consideraciones Generales}

Consideramos, con Garvin, que las hipótesis en sentido técnico son en mayor medida parte del aparato metodológico, y no del aparato teórico, ya que ellas

3 Los textos Análisis Gramatical (Pike y Pike, 1991) y Conceptos Lingüísticos (Pike, 1995) presentan una versión más actualizada de la teoría tagmémica. En esta segunda versión -a diferencia de la versión clásica que presenta en las fórmulas tagmémicas sólo los casilleros de función gramatical y clase instanciadora- se proponen cuadros operativos de cuatro casillas: posición, función, clase y cohesión. En nuestra investigación hemos seguido los postulados de la primera versión de la teoría, pues estimamos que ésta da cuenta de manera satisfactoria de los fenómenos que intenta describir.

4 Por razones de espacio, en este artículo sólo apuntamos algunos de los aspectos más importantes de la tagmémica. Para una revisión más completa de este marco de referencia, el lector puede consultar la tesis citada, donde se aborda con considerable grado de detalle el modelo tagmémico y se justifica con abundante ejemplificación la pertinencia de éste para el análisis de una lengua como la que nos ocupa. 
emergen durante el proceso de aplicación de un método, sin ser parte de los fundamentos teóricos de la investigación (Garvin, 1979: 112). De esta forma, desde la estrategia metodológica se derivará un gran número de hipótesis de "pequeña escala" que deberán ser siempre contrastadas con los nuevos datos obtenidos en el curso de la investigación.

Desde la perspectiva del Empiricismo Funcional, Garvin concibe las manifestaciones lingüísticas como objetos estructurados; dicha estructura es susceptible de ser descubierta a través del análisis. En otras palabras, el objetivo del análisis consiste en descubrir la estructura que subyace a los datos, conduciendo últimamente a una descripción en la cual éstos estén incorporados en sus dimensiones estructuralmente relevantes.

\subsection{Los Datos}

Los datos utilizados en esta investigación provienen principalmente de cuatro fuentes:

a) Listas léxicas elicitadas por Salamanca (2002). A partir de los datos elicitados en estas listas, especialmente los relativos a palabras de objetos, fue posible plantear las primeras hipótesis en cuanto a las marcas de género y número de los adjetivos en general, además de su estrecha relación de concordancia con el sustantivo. Los siguientes son algunos ejemplos del tipo de información requerida y la respuesta de los informantes:

Input en español (contraste de género y número) respuesta en rromané el muchacho grande la muchacha grande los muchachos grandes las muchachas grandes o rakló baró e raklí barí e raklé baré e rakliá baré

En estos enunciados se pueden apreciar, a través del contraste, las marcas de género y número en los adjetivos calificativos: el morfema de género masculino "-ó", el morfema de género femenino "-î" y el morfema de número plural "-é". Es interesante notar que, en el adjetivo, el morfema de pluralidad no co-ocurre con los morfemas de género. De paso, podemos llamar la atención en cuanto a la productividad de este tipo de método de elicitación, pues con un reducido número de entradas ya es posible postular las primeras hipótesis respecto de la morfología flexiva del adjetivo.

b) Textos orales. Otra de las fuentes para el análisis está dada por tres relatos tradicionales registrados por Salamanca (2002). Estos textos se encuentran transcritos fonológicamente y presentan una segmentación morfológica, ésta última de gran utilidad para nuestra investigación. Este tipo de corpus presenta la ventaja de no estar predispuesto por el investigador, lo que hace factible que aparezcan datos no previstos inicialmente. De esta fuente se obtienen datos relevantes, como los siguientes: 


\begin{tabular}{|c|c|c|c|c|}
\hline múrrn- & $-i$ & čar- & $-i \tilde{c}$ & $-a$ \\
\hline adj. pos. 1 sing. & fem. & carpa & dim. atem ${ }^{5}$. & fem. \\
\hline
\end{tabular}

\begin{tabular}{|c|c|c|c|c|c|c|c|}
\hline$k a j$ & $m u k-$ & $-l$ & -ém & l- & $-a$ & nev- & -orr \\
\hline pron, rel. & dejar & p.perf. & 1 sing. & pron. acus. 3 & fem. & nuevo & dim. tem. \\
\hline
\end{tabular}

que nuevita la dejé

$\begin{array}{lcccccccc}\text { mala }(d)- & -d & -e ́ m & l- & -a & \text { phur- } & -a n & -i & \rightarrow l \\ \text { encontrar } & \text { p. perf. } & 1 \text { sing. } & \text { pron. acus. } 3 & \text { fem. } & \text { viejo } & \text { aum. } & \text { fem. }\end{array}$

la encontré muy vieja,

donde encontramos, por una parte, el adjetivo posesivo de primera persona ("múrrni") y, por otra, observamos que los adjetivos calificativos reciben sufijos derivacionales diminutivos y aumentativos (-orr y -an, respectivamente), previos a la flexión.

c) Pasajes del Nuevo Testamento Romané (Sociedad Bíblica Chilena, 2007). La traducción al rromané del Nuevo Testamento, si bien no se trata de un trabajo que considere en algún sentido fines lingüísticos, sino evangelizadores, constituye un corpus abundante para el análisis que hemos llevado a cabo en esta investigación. Los datos de este texto fueron cotejados con los obtenidos directamente de los hablantes a través de sus relatos tradicionales.

El siguiente es un ejemplo de un enunciado tomado de este texto:

O villelo chachuno [...] avela pe gava sueto (Sociedad Bíblica Chilena, 2007: 202)

Esa luz verdadera, vino a este mundo

Aquí podemos descubrir el adjetivo demostrativo gavá, que indica cercanía respecto del hablante y posee género masculino y número singular.

\subsection{Los Informantes}

Para la elicitación de los cuentos tradicionales y posterior chequeo de la información se consideraron diez informantes, cantidad que se estima apropiada para este tipo de investigaciones. En efecto, la cantidad de informantes es una sugerencia que se somete a las condiciones del trabajo de campo. En palabras de Salas: "El número de [...] encuestados es cuestión empírica, de modo que sólo puede ser determinado por medios empíricos, o sea, una vez que el proyecto está en curso, a la vista de la homogeneidad/heterogeneidad de la conducta [...] observada" (Salas, 1992: 3). 
Para la selección de los informantes, se consideraron los principios clásicos para este tipo de trabajos (tomados de Samarin, 1967: 61-64):

Edad: colaboraron gitanos cuyas edades fluctuaban entre los dieciséis y cincuenta años, pues en este rango el sistema lingüístico ya está asentado y hay escasas probabilidades de encontrar un deterioro en las habilidades cognitivas.

Sexo: se trabajó preferentemente con informantes de sexo masculino. Esta decisión se explica por el alto grado de aprensión que existe en la cultura gitana por el contacto entre las mujeres de este grupo con hombres no gitanos.

Conocimiento de la lengua de contacto: se privilegió la colaboración de hablantes que manifestaban un dominio fluido de ambas lenguas.

\subsection{Morfemas y método de descubrimiento}

En nuestro trabajo, partimos de la siguiente premisa metodológica: "Analysis must go hand in hand with field work" (Nida, 1946: 192). Como se señaló con anterioridad, las primeras hipótesis acerca de la estructura de la lengua surgen a partir del acercamiento con los datos; por su parte, tales hipótesis son contrastadas con nuevos datos, los cuales permiten afirmarlas o refutarlas.

En un trabajo como el que presentamos, la identificación de morfemas es una tarea fundamental. Elson y Pickett, siguiendo a Hockett, definen el morfema como "...the smallest individually meaningful elements in the utterances of a language." (Elson y Pickett, 1964: 7).

De acuerdo con los mismos autores, el proceso de descubrimiento de los morfemas

$[\ldots]$ is a process of substitution and comparing recurring partials. Two o more utterances partly alike but partly different are compared. The like parts, if they have the same meanings, are recurring partials. These partials constitute a point of reference which allows us to compare the unlike parts. (Op. Cit.: 8)

Así, por ejemplo, en el rromané jorajané tenemos los siguientes enunciados:

$$
\begin{array}{ll}
\text { kalí } & \text { 'negra' } \\
\text { phurí } & \text { 'vieja' }
\end{array}
$$

Observando estos datos, reconocemos un parcial recurrente "-î" que señala género femenino.

Un ejemplo de determinación de morfemas por vía del contraste se aprecia en los siguientes enunciados:

$$
\begin{array}{ll}
\text { yek phurí 'una vieja' } \\
\text { yek phuró 'un viejo' }
\end{array}
$$


El procedimiento más utilizado en este artículo para la detección de morfemas es el contraste.

\subsection{Observaciones distribucionales}

- Orden de las clases de morfemas: Se indica aquí cuál es el orden relativo de los morfemas y si algunos morfemas son mutuamente obligatorios o excluyentes.

- Tipo de alternancia morfológica: Aquí se precisa si los alomorfos están fonológicamente condicionados o la forma fonológica parece ser completamente arbitraria y, por lo tanto, supletiva o léxica.

Es posible proponer que algunos alomorfos de las raíces ${ }^{5}$ y afijos que componen los adjetivos resultan de procesos morfofonémicos, tales como la supresión, la epéntesis o la metátesis. Así, por ejemplo, se ha determinado que la no ocurrencia del morfema que indica género en los calificativos, en su forma plural, se debe a un proceso de supresión para evitar secuencias no aceptables para el rromané.

\section{Análisis Morfológico}

\subsection{Notas sobre la clasificación de los demostrativos}

En la tradición gramatical española, la clasificación de los demostrativos ha ido en dos direcciones: considerarlos pronombres, destacando su capacidad de cumplir las funciones propias del sustantivo, y clasificarlos como adjetivos. Partidario de la primera opción es Andrés Bello, para quien los "pronombres demostrativos son aquellos de que nos servimos para mostrar los objetos señalando su situación respecto de determinada persona." (Bello, 1988[1847]: 270). En esta misma línea se inscriben los planteamientos de Salvador Fernández, en cuya gramática se definen los demostrativos como: "pronombres que en español realizan el señalamiento al campo deíctico" (Fernández, 1951: 236). Pero, aunque los agrupa dentro de los pronombres, Fernández sostiene que los demostrativos pueden funcionar como término primario o secundario, reconociendo el valor adjetivo de este último funcionamiento: "Los (demostrativos) adjetivos realizan, en general, las mismas funciones que el artículo, como términos secundarios tanto del nombre común como del nombre propio o del nombre de cosa única" (Ibíd.: 240). Algo similar encontramos en la gramática de Marcos Marín, quien sitúa los demostrativos dentro de la clase de los pronombres, señalando que pueden cumplir tanto la función primaria como la secundaria, caso en el cual

5 A lo largo de nuestro trabajo, la noción "raíz" tiene un uso aplicable no sólo a aquellas formas que portan un contenido con algún correlato referencial, sino que se aplica también a aquellas formas susceptibles de constituir el soporte a partir del cual se despliegan los morfemas derivativos o flexivos. Esto nos permite hablar de "raíces" en clases de palabras como los adjetivos demostrativos y posesivos. 
los "demostrativos se pueden entender como actualizadores de los sustantivos a los que acompañan." (Marcos, 1998: 167).

En Alarcos encontramos la opción por categorizar los demostrativos como adjetivos, específicamente dentro de la clase de los determinantes. Para este autor es innecesaria la distinción entre adjetivos y pronombres demostrativos, pues "todos los adjetivos, mediante la sustantivación, son capaces de cumplir en el enunciado la función de sustantivos." (Alarcos, 1994: 89).

Algo en lo que coinciden las gramáticas revisadas es en el valor deíctico de los demostrativos: estas unidades funcionan como señaladores en relación con un punto de referencia. Ahora bien, tal punto de referencia puede ser de distinta naturaleza, según forme parte de distintos campos de señalamiento. Esto último justifica la tradicional distinción entre "la llamada deixis, en virtud de la cual el pronombre señala de una manera directa objetos que se encuentran dentro de nuestro horizonte sensible y la llamada anáfora, en virtud de la cual reproducimos o señalamos lo que ha sido nombrado recientemente en el discurso." (Fernández, 1951: 241). De este modo, la deixis propiamente tal, la llamada "demonstratio ad oculos", se da en el contexto de un campo de señalamiento demostrativo, a diferencia de la anáfora, que se produce en el campo del contexto o campo sintáctico (la que, siguiendo siempre a Fernández, da lugar a una suerte de "demonstratio reflexiva"). Traemos a colación lo anterior, pues la distinción establecida nos permite acotar nuestra investigación en las formas que permiten la deixis mostrativa, la demonstratio ad oculos, en rromané.

A pesar de que nuestra investigación es estrictamente morfológica y, como apuntamos antes, se sitúa en el nivel de la palabra, es inevitable (y justificado) hacer alusión a otros niveles, al menos para delimitar los usos de las formas que nos ocupan.

\subsection{Demostrativos en el rromané jorajané ${ }^{6}$}

Los demostrativos, dentro de la categoría de los adjetivos, constituyen en rromané un subsistema finito y altamente simétrico. A diferencia del español, que muestra distintos grados de proximidad respecto de la $1^{\mathrm{a}}$ persona gramatical (este, etc.), la $2^{\mathrm{a}}$ (ese, etc.) y el apartamiento de ambas (aquel, etc.) $)^{7}$, en rromané sólo encontramos dos distinciones formales de demostrativos, tal como se puede apreciar en los siguientes ejemplos:

1. Gavá chavrró Este muchacho
5. Galá minonorré Estos pequeñitos

6 En la presente investigación hemos limitado nuestra atención en las formas del caso nominativo.

7 En esta descripción de los demostrativos, seguimos a Carratalá (1980: 238). Valga mencionar lo anterior, pues en otros autores (por ejemplo, Alarcos, 1994: 92) encontramos que "aquel" señala a la "no-persona", la $3^{a}$ persona gramatical. 
2. Gua (Gová) chavrró

Ese muchacho

3. Gayá riat

Esta noche

4. Goyá riat

Esa noche
6. Golá minonorré

Esos pequeñitos

7. Galá esgóde

Estas cosas

8. Golá esgóde

Esas cosas

Así, la deixis - el señalamiento mostrativo-en esta lengua tendría dos valores, expresados mediante sus demostrativos: proximidad y alejamiento.

\subsubsection{Formas}

En los siguientes cuadros se presenta el conjunto completo de demostrativos de la lengua gitana:

\section{Proximidad}

\begin{tabular}{|c|c|c|}
\cline { 2 - 3 } \multicolumn{1}{c|}{} & Masculino & Femenino \\
\hline Singular & Gavá & Gayá \\
\hline Plural & Galá & Galá \\
\hline
\end{tabular}

\section{Alejamiento}

\begin{tabular}{|c|c|c|}
\cline { 2 - 3 } \multicolumn{1}{c|}{} & Masculino & Femenino \\
\hline Singular & Guá & Goyá \\
\hline Plural & Golá & Golá \\
\hline
\end{tabular}

\section{Observaciones}

En el cuadro se puede apreciar que todas las formas se vinculan con valores de género masculino o femenino, es decir, que en la lengua gitana no existen formas neutras para el demostrativo.

Por otra parte, como se observa en el esquema, la simetría del conjunto es casi perfecta, excepto por el demostrativo de alejamiento masculino singular: guá. No obstante, la aparente irregularidad de este elemento desaparece si postulamos que esta forma es una contracción de un demostrativo posible gová, en el que se produce la supresión de la aproximante labial y una diptongación (mediante el ascenso de la vocal "o" a la altura de "u"). Respecto de esto último, es importante señalar que ambos procesos no son excepcionales ni privativos del rromané, pues, por ejemplo, se observan también en español (aunque más asociados al habla subestándar). En efecto, es recurrente en ciertas variaciones sociolectales

8 Cuando el sustantivo al que este demostrativo determina, finaliza en vocal tónica "-é", puede producirse una armonía vocálica y este puede asumir la forma "golé". Es el caso en "Golé llené 'esas gentes'. 
del español elidir completamente la consonante labial, lo que sucede en palabras como "nueve" o "subir" (que devienen en "nuee" o "suír", respectivamente); o diptongar una secuencia de vocales abiertas, como en la palabra "coagularse" (que deviene en una pronunciación semejante a "cuagularse"). De ahí que la propuesta de un demostrativo subyacente "gová" nos parezca respaldada por evidencia interna y externa al rromané ${ }^{9}$.

Por su parte, podemos notar que -tal como ocurría con los calificativos- las formas plurales no presentan marcas de género, lo que da cuenta de la consistencia interna del sistema de adjetivos del rromané.

Establecer a ciencia cierta el valor deíctico de estos demostrativos es algo que requeriría atención especial: dicha proximidad y alejamiento podrían establecerse en función de la primera persona gramatical o en función de ambas (los sujetos del coloquio), o bien, se podrían postular dos grados de cercanía: uno respecto de la primera persona y uno respecto de la segunda. Se trata de un asunto que difícilmente podríamos solucionar en un trabajo descriptivo como el nuestro, sobre todo por los alcances lingüístico-cognitivos del problema. Asumiendo esta limitación, nos parece que establecer los valores de "proximidad" y "alejamiento" configura una imagen de la deixis que resulta intuitiva y apropiada para el estado actual de la investigación en torno a la lengua gitana. Dicho sea de paso, algunos autores sostienen que en español, pese a la distinción formal tripartita del señalamiento establecida por los demostrativos, el valor de la demostración no deja de ser binario: se distingue en último término proximidad y alejamiento.

Como se señaló anteriormente (en 6.1), nuestra atención sobre las formas demostrativas se ha centrado en expresiones que implican señalamiento espacial, la llamada "demonstratio ad oculos". Una pesquisa más amplia podría dar cuenta de formas de señalamiento textual en esta lengua, manifestado esto en recursos anafóricos o catafóricos.

\subsubsection{Segmentación morfológica}

En el siguiente esquema, presentamos nuestra propuesta segmentación morfológica de los demostrativos del rromané jorajané:

9 Una opción analítica ligeramente distinta consiste en postular una forma subyacente "gooá" (con la forma discontinua típica "g-...-a", que indica "demostrativo"; el morfema señalador de distanciamiento "-o-"; y la forma subyacente de género masculino "-o-"), donde la co-ocurrencia de las dos vocales "o" se reduce a una forma única que da como resultado la forma teórica "goá". Desde esta forma bisilábica es posible derivar -por ascenso de la vocal "o" y consecuente transformación en la semiconsonate "w"- una secuencia vocálica en diptongo que da lugar a la forma "gwa". Ahora, dada la frecuencia con que esta palabra ocurre precedida de vocal, con el consiguiente relajamiento de la consonante inicial, es posible derivar una palabra monosilábica, iniciada por una semiconsonante labializada "w", más la vocal "a". Esto sería comparable al paso de la preposición "para", a la forma coloquial "pa". Indudablemente, tanto "wa como "pa", constituyen, desde el punto de vista de la platilla silábica, la estructura más óptima: $\mathrm{CV}$. 


\begin{tabular}{|c|c|c|c|}
\hline & no (sing.) & Femenino (sing.) & \\
\hline & g... - a - v- $\varnothing-\ldots a ́$ & g... - a - y $-\varnothing-\ldots a ́$ & g... - a $-\varnothing-1-\ldots a ́$ \\
\hline Anart & g... - o-v- $\varnothing-\ldots a ́$ & 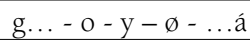 & g... - o- $\varnothing-1-\ldots a ́$ \\
\hline
\end{tabular}

\section{Observaciones}

Para la segmentación morfológica de los demostrativos, hemos optado por proponer una raíz discontinua "g-.......á". Flanqueados por esta raíz, se presentan -de acuerdo con nuestra interpretación- el morfema de distancia y la flexión de género y número, con sus respectivos morfemas instanciadores.

Así:

\begin{tabular}{|c|c|c|c|c|}
\hline \multicolumn{4}{|c|}{ Raíz } & \\
\hline & Distancia & Género & Número & \\
\hline g... & $\begin{array}{l}\text { morf. de } \\
\text { distancia }\end{array}$ & morf. de género & morf. de número & …á \\
\hline
\end{tabular}

Esta fórmula se puede completar con una forma concreta, por ejemplo "gavá", con lo que obtenemos el siguiente esquema:

\begin{tabular}{|c|c|c|c|c|}
\hline \multicolumn{4}{|c|}{ Raíz } \\
\cline { 2 - 4 } & Proximidad & Masculino & Singular & \\
\hline $\mathrm{g} \ldots$ & $\mathrm{a}$ & $\mathrm{v}$ & $\varnothing$ & $\ldots \mathrm{a}$ \\
\hline
\end{tabular}

Utilizar la noción de "morfema discontinuo" permite, a nuestro juicio, dar cuenta de manera consistente de la ocurrencia sistemática de las formas "g-" y "-á" en toda la serie de los demostrativos. De acuerdo con esta interpretación, a estos elementos les corresponde el valor/significado de demostrativo. Otra opción consistiría en interpretar la vocal final "-á” como parte de la desinencia, lo que nos impondría (1) darle estatus de morfema y, por lo tanto, asignarle (ciertamente de manera forzosa) algún valor distinto de los otorgados a los demás morfemas aislados o (2) otorgarle un valor puramente fónico. Ambas opciones nos parecen extremos no apropiados para un análisis morfológico que, evitando caer en la sobreinterpretación, procura dar cuenta de los vínculos entre forma y contenido (sean éstos léxicos o gramaticales).

\subsubsection{Asignación de morfos a morfemas}

\section{Género}

$\{-\mathrm{v}-\}$ 'masculino'. Este morfema tiene dos alomorfos: "-ø-", en el plural, y "-v-" en otros casos.

\{-y-\} 'femenino'. También presenta dos alomorfos: “-ø-", en el plural, e "-y-" en otros casos.

En ambos casos, se trata de alternancia entre alomorfos morfológicamente condicionados. 


\section{Número}

$\{-\phi-\}$ 'singular'

$\{-1-\}$ 'plural'

\section{Distancia}

$\{-a-\}$ 'proximidad'

$\{-o-\}$ 'alejamiento'

\section{Observaciones}

Los procesos de supresión en el rromané son frecuentes, ya sea por condicionamientos fonológicos o morfofonémicos (Cfr. Salamanca, 2002). En el caso de las unidades que nos ocupan, es posible postular que en los demostrativos plurales -"galá" y "golá"- se produce la supresión de la marca de género por un condicionamiento fonémico (más específicamente, fonotáctico) que busca evitar secuencias de fonemas poco habituales en esta lengua.

Con matices, para los que procuraremos algún tipo de plausibilidad morfofonémica, es posible postular que las formas instanciadoras de género que presentan los demostrativos en singular concuerdan con las marcas que expresan el género en las otras formas nominales (Cfr. Salamanca, 2002), a saber, "-ó" e "-î", para masculino y femenino, respectivamente. Como se hizo evidente en la segmentación morfológica, la marca de femenino en los demostrativos es "-y-", una semiconsonante que por su propia naturaleza es átona. Lo anterior es precisamente uno de esos matices mencionados al principio del párrafo: la tonicidad de la marca de femenino, característica de los sustantivos (chorr-í 'hija'; rrom-í 'gitana', por ejemplo), se ve afectada por el contexto fónico, pues la forma en cuestión se presenta entre vocales, lo que -de mantenerse la forma tónica- generaría secuencias vocálicas (como "aía" y "oía") que entran en colisión con las estructuras silábicas habituales de la lengua ${ }^{10}$. Postulamos, entonces, que para evitar una secuencia no posible en la lengua y/o derivar una secuencia más óptima, se produce una consonantización de la vocal, lo que genera una palabra (un disílabo) del tipo $\mathrm{CVCV}^{11}$.

Siguiendo con nuestra hipótesis de identidad en las marcas de género entre los demostrativos y los nominales, nos parece apropiado postular un proceso similar al anterior para la marca de género masculino "-ó". En efecto, si tomamos, por ejemplo, como referencia la forma "gavá", podemos postular que en un nivel de abstracción mayor la forma subyacente a ella es "gaoá", sólo que, por incluir ésta una secuencia vocálica no compatible con las estructuras silábicas propias del rromané y ser una forma poco frecuente en las lenguas en

10 Los tipos de sílaba posibles en rromané jorajané se describen en Salamanca (2002: 127-128).

11 Esta tendencia hacia secuencias más simples se sustenta en la asunción de que "[...] CV is the universal syllable type [...]" (Burquest, 2001: 153). 
general, se recurre a una acomodación fonológica que genera una palabra cuya plantilla silábica es CVCV. Esta estructura, como hemos dicho, se considera óptima no sólo desde el punto de vista de la lengua que nos ocupa, sino de las lenguas en general.

En relación con el morfema de número, la lengua rromané sigue la tendencia de la lenguas indoeuropeas en cuanto a la ausencia de marcas formales para el número singular.

La ausencia de alomorfía en el plural, contenido marcado por una consonante invariable "-l-", es un asunto destacable, en la medida que reafirma la hipótesis de que los procesos de supresión son un recurso que la lengua utiliza para evitar secuencias vocálicas anómalas o atípicas. Así, lográndose la estructura CVCV (vía supresión de la marca de género) se hace innecesaria la supresión del morfema de número o su variación alomórfica.

Es posible establecer, por último, un interesante vínculo entre el español y el rromané en cuanto a la relación, sobre la que llama la atención E. Carratalá, entre los demostrativos y los puntualizadores demostrativos. Siguiendo a este autor, ambas categorías léxicas se encuentran históricamente emparentadas ${ }^{12}$. Dichos puntualizadores se presentan en el siguiente cuadro (tomado de Carratalá, 1980: 240):

\begin{tabular}{|c|c|c|c|}
\hline \multicolumn{4}{|c|}{ Demonstratio ad oculos } \\
\hline Lugar & Estática & Persona & Dinámica \\
\hline \multirow{2}{*}{ Próximo } & a-qu-í & $1^{\mathrm{a}}$ & a-c-á \\
& a-hø-1́ & $2^{\mathrm{a}}$ & --- \\
\hline Apartado & a-ll-í & $1^{\mathrm{a}} \mathrm{y} 2^{\mathrm{a}}$ & a-ll-á \\
\hline
\end{tabular}

Lo interesante aquí es que en rromané existe un par de puntualizadores demostrativos que no sólo establecen un tipo de señalamiento espacial similar al de los demostrativos, sino que, además, presentan con éstos una semejanza formal difícilmente atribuible a una mera homofonía. Obsérvense los siguientes ejemplos:

\section{katé si mo chavrró \\ aquí está mi hijo \\ koté si mo chavrró \\ allí está mi hijo}

Nótese que la única diferencia entre estos dos puntualizadotes demostrativos es la presencia de la vocal "a" en el puntualizador demostrativo de proximidad y "o" en el de alejamiento. En esto está precisamente la semejanza formal men-

12 Marcos Marín llama la atención sobre estas formas puntualizadoras, en relación con los demostrativos, ya que tienen también un valor deíctico, pero es claro en señalar que se trata de palabras de naturaleza categorial adverbial que, al no tener flexión de género y número "no funcionan como estas formas demostrativas que pueden desempeñar las funciones primaria y secundaria" (Marcos, 1998: 167). 
cionada en el párrafo precedente, pues "a" y "o" son las formas que en nuestro análisis hemos segmentado como los morfemas indicadores de los valores de 'proximidad' y 'alejamiento' en los adjetivos demostrativos. Podemos considerar, como un elemento más para esta relación, que así como en español existe un puntualizador equivalente a cada demostrativo (aqui, ahi y alli; para este, ese y aquel, respectivamente), en rromané existe también esta equivalencia, pues los puntualizadores katé y koté se correlacionan con los demostrativos gavá y gová (gua), respectivamente. Claro que, en rromané, la relación entre estas formas y contenidos es aún mayor, puesto que incluso coinciden en las formas fónicas que marcan proximidad y alejamiento ("a" y "o", respectivamente).

\section{Consideración final}

De todo lo dicho, se derivan conclusiones respecto de esta clase de adjetivos, las cuales convendría explicitar aquí. Sin embargo, a fin de dar una perspectiva global del tratamiento de los demostrativos y posesivos como conjunto, las hemos diferido a las conclusiones generales del segundo artículo y final.

\section{Bibliografía}

Alarcos, E. (1994). Gramática de la Lengua Española, Espasa Calpe: Madrid.

Bello, A. (1988[1847]). Gramática de la Lengua Castellana. Madrid: Arco Libros.

Bursquest, D. (2001). Phonological Análisis: A functional approach. Dallas: Summer Institute of Linguistics.

Carratalá, E. (1980). Morfosintaxis Del Castellano Actual. Barcelona: Labor.

Elson, B. y V. Pickett. (1964).

An Introduction to Morphology and Sintax. Ciudad de México: Summer Institute Linguistics.

Elson, B. y V. Pickett. (1980). Beginning Morfhology and Syntax. Ciudad de México: Summer Institute of Linguistics.

Fernández, S. (1951). Gramática Española. Madrid: Revista de Occidente.

Franzese, S. (1997). "Il dialecto dei Rom Xoraxané, note grammaticali", en página electrónica de O Vurdon, URL: <http: //www.vurdon.it/03. htm.

Garvin, P. (1979). «Un epistemología empiricista para la lingüística», en Revista de Lingüística Teórica y Aplicada Vol. 17, pp 109-127.

González A. y Salamanca, G. (2001).

«Descripción fonológica del romané de Chile», en Revista de Filología y Lingüística de la Universidad de Costa Rica Vol. XXVIII(1), pp. 177-197.

Grant, A. (1994). Romani: A Conspectus. Notes o Romani Lenguaje and Linguistics. Bradford: University of Bradfor. 
Morfología de los Adjetivos Demostrativos en el / Dr. @ Diego Lizarralde y Dr. Gastón Salamanca

Hancock, I. (1993). Grammar of Vlax Romani. Texas: Romanestan Publications.

Marcos Marín, F. et all. (1998).

Gramática Española. Madrid: Síntesis.

Nida, E. (1946). Morphology: The descriptive análisis of de words. Michigan: The University of Michigan Press.

Pike, K. (1995). Conceptos lingüisticos. Dallas: Summer Institute of Linguistics.

Pike, K. y E. Pike. (1995). Análisis Gramátical. Ciudad de México: Universidad Nacional Autónoma de México.

Salamanca, G. (2002). Morfología nominal y verbal del romané, lengua de los gitanos de Chile, Tesis para optar al grado de Doctor en Lingüística, Universidad de Concepción.

Salamanca, G. (2004a). «Los gitanos y su lengua», en Revista de Lingüística Teórica y Aplicada Vol. 42 (1), pp. 63-85.

Salamanca, G. (2004b). «Notas sobre la morfología verbal del rromané, lengua de los gitanos de Chile (1)», en Logos $\mathrm{N}^{\circ}$ 14, pp. 73-96.

Salamanca, G. (2005). «Vitalidad lingüística y cultural en un mundo globalizado: El caso del rromané, frente a las lenguas vernáculas chilenas», en Sociedad Hoy No 8-9.

Salamanca, G. (2006). «Notas sobre la morfología nominal del rromané, lengua de los gitanos de Chile (II)», en Alpha No 22, pp. 209-222.

Salamanca, G. (2008). «Notas sobre la morfología nominal del rromané, lengua de los gitanos de Chile (I)», en Literatura y Lingüística $\mathrm{N}^{\circ} 19$, pp. 211-233.

Salamanca, G. y González, A. (1999). "Gitanos de Chile: Un acercamiento etnolingüística", en Atenea $\mathrm{N}^{\circ} 480$, pp. 141-172.

Salamanca, G. y Lizarralde, D. (2008). «Propuesta de un grafemario para el rromané jorajané, lengua hablada por los gitanos de Chile (1)», en Universum $\mathrm{N}^{\circ}$ 23(1), pp. 226-247.

Salas, A. (1992). $\quad$ "Fonología del aymara hablado por la población infantil de la Provincia de Parinacota, Ms. Inédito.

Samarin, W. (1967). Field Linguistics: A Guide to Linguistic Field Work. New York: Holt, Rinehart and Wiston.

Sociedad Bíblica Chilena. (2007). Nuevo Testamento Romané. Brasil: Sociedades Bíblicas Unidas.

Wagner, C. et all). (1978). "Glosario de términos lingüísticos", en Documentos Lingüísticos y Literarios. No 2. Universidad Austral. 